\title{
Perinatal Mental Health: One of the Biggest Challenges in Coronavirus Disease-19 Crisis
}

\author{
Goce Kalcev $^{1 *}$, Antonio Preti ${ }^{1}$, Germano Orrù ${ }^{2}$, Mauro Giovanni Carta ${ }^{1}$ \\ ${ }^{1}$ Department of Public Health, Clinical and Molecular Medicine, University of Cagliari, Cagliari, Italy; ${ }^{2}$ Department of Surgical \\ Sciences, University of Cagliari, Italy
}

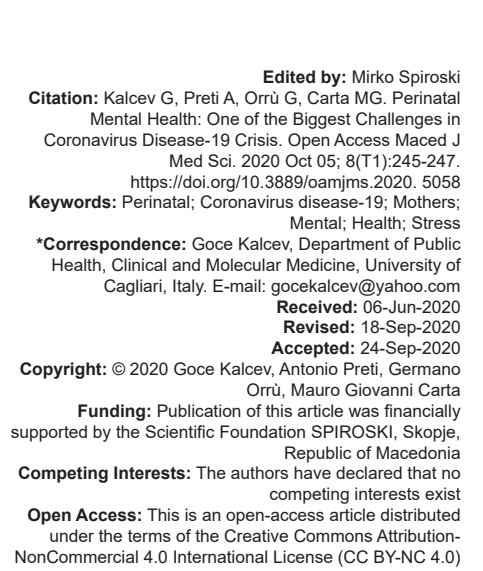

\section{Abstract}

Coronavirus disease (COVID)-19 epidemic is currently conceived as one of the major factors for stress and anxiety for pregnant women around the world. Stress, especially in early pregnancy, is a risk factor for preterm birth. The negative impact of quarantine on mental health in pregnant women should also be taken into account. A large number of benefits of breastfeeding for the mental and physical well-being of both mother and newborn outweigh the potential risks of COVID-19-related transmission and disease. Prenatal and postnatal mental health should be prioritized in facing the current ongoing pandemic.
With the spread of the coronavirus disease (COVID)-19, there has been widespread concern about the possibility of its intrauterine transmission from the mother to the fetus during the pregnancy [1]. As a result, dilemmas for the morbidity and mortality rate in mothers and newborns have arisen together with the complications that would occur during pregnancy or after that. The lack of evidence-based information on these issues among their enormous importance is a source of stress for pregnant women and future mothers [2], [3]. Nowadays, the severe acute respiratory syndrome-CoV-2 epidemic is conceived as one of the major factors for stress and anxiety for pregnant women around the world [4].

One of the biggest problems we want to focus on is the practice of routinely separating the mother from her newborn after birth as a measure against the transmission of the COVID-19 virus. According to research conducted in China, this strategy, apart from stopping breastfeeding, also has huge consequences for the mother's mental health in the form of feelings of shame, self-blame, and the onset of depression [5]. The practice of stopping breastfeeding should be actively avoided. Consequently, proper care and support measures should be taken to reduce or at least mitigate the negative effects. In every socioeconomic position, breastfeeding raises survival and delivers lifelong health and progress advantages to newborns and infants. The transmission of COVID19 across breast milk and breastfeeding has not been observed. In the group of few cases of confirmed COVID-19 infection in children from other origins, most have confronted only mild or asymptomatic form. During breastfeeding, a mother should carry out proper hygiene measures, involving wearing a medical mask if available, to decrease the possibility of droplets with COVID-19 being expanded to her infant. No active COVID-19 virus has been confirmed in the breast milk of any mother confirmed/suspected to have COVID-19. It is unexpected that the virus can be transferred by a mother with confirmed/suspected COVID-19 virus through breast milk [6]. The message of the authors on this issue is that a large number of benefits of breastfeeding for the mental and physical well-being of both mother and newborn outweigh the potential risks of COVID-19-related transmission and disease.

Within this framework, how to protect the mental health of a pregnant woman from the short- and long-term consequences of this actual pandemic? A lot of pregnant women live in poor households together with other people in a limited space, caring for their 
other children or for elderly people at the same time. In the current pandemic, this may impact on the mental health of pregnant women. According to two of the Millennium Development Goals (four and five), overall health cannot be ensured without mental health [7]. Stress, especially in early pregnancy, is a risk factor for preterm birth [8]. The development and implementation of dedicated mental health services are urgent for this special category of the population. Anxiety and stress in pregnant women are responsible for consequences such as preeclampsia, depression, increased nausea and vomiting during pregnancy, preterm labor, low birth weight, and low appearance, pulse, grimace, activity, and respiration score [9].

Moreover, many pregnant women currently are not visiting a doctor for fear of contagion with COVID-19. Travel bans and the thought of not having a doctor available every time are especially frightening for a pregnant woman. Both situations pose a huge threat to a pregnant woman's mental health. Providing advice and support through telecommunications is of particular importance. In addition, depression is frequent during pregnancy, affecting up to $28 \%$ during late pregnancy [10].

The impact of the quarantine lockdown on the mental health of pregnant women should be not overlooked. In general, quarantine, self-isolation, and loneliness have a negative impact on mental health, resulting in increased levels of stress, anxiety, depression, and self-harm, but not only for pregnant women but also for the entire population [11], [12]. The fear that comes with it may represent an additional source of stress that could negatively affect a mother's pregnancy. The quarantine will be probably responsible for the worsening of pre-existing mental disorders and may also worsen mental stress in future mothers with non-psychiatric disabilities due to the reduced medical care and support during the pregnancy. According to the data received from one Canadian study, pregnant women described significant levels of psychological distress in form of pregnancy-specific anxiety symptoms (68\%), general anxiety (57\%), and as depression (37\%). In comparison with the findings from the previous community pregnancy cohorts, these symptoms are considerably higher [13]. Another study among Turkish pregnant women showed increased scores on screening instruments for depression and anxiety during this pandemic [14]. As stated by an Italian survey, the psychological impact of the COVID-19 is classified as serious by $53 \%$ of pregnant women. In addition, two-thirds of the respondents experienced higher levels of anxiety than normal [15]. Clinical care of pregnant women with COVID-19 depends on the severity of the disease. Most (86\%) of the pregnant with known or suspected COVID-19 have mild symptoms (no shortness of breath) that now require hospital-level care, while there are no obstetric problems. However, they should be followed and monitored for eventually progression [16].
It is necessary for health professionals to create and disseminate guidelines for mental health for vulnerable categories. What's more significant, measures should be taken by the government, community, and relevant departments to provide timelyspecific psychiatric services for pregnant women. Already in February 2020, some reports published in The Lancet indicated that the care of the mental care of mothers is vital [17]. The authors considered that it is crucial for pregnant women and mothers to be in constant contact with reliable and confidential information about the course of the COVID-19disease, prevention, and its impact on the perinatal and postnatal period [6]. By contrast, receiving inappropriate and unverified information can increase stress and fears [18]. Communication with pregnant women and mothers should be shown empathy, understanding of their needs, feelings, and support for expressing their emotions. Moreover, support from loved ones to identify positive strategies for dealing with their emotions and stress plays an essential role [6]. On the other hand, fathers play an important role in pregnancy. Supporting from the fathers during this crisis is an essential step in preserving the mental health and well-being of pregnant women and mothers. Maintaining contact with the environment through telecommunications and social networks is one of the principal ways to meet the needs that provide welfare. Understanding the needs of mothers and fathers will lead to a faster and better response to future unexpected events. To sum up, prenatal and postnatal mental health should be prioritized in facing the current ongoing pandemic [19].

\section{Acknowledgments}

Goce Kalcev (MD) was participating in the writing of this paper in the framework of the International $\mathrm{PhD}$ in Innovation Sciences and Technologies at the University of Cagliari, Italy.

\section{References}

1. Qiao J. What are the risks of COVID-19 infection in pregnant women? Lancet 2020;395:760-2. https://doi.org/10.1016/ s0140-6736(20)30365-2

2. Mardani M, Pourkaveh B. A controversial debate: Vertical transmission of COVID-19 in pregnancy. Arch Clin Infect Dis 2020;15(1):e102286. https://doi.org/10.5812/archcid.102286

3. Liu D, Li L, Wu X, Zheng D, Wang J, Liang B, et al. Pregnancy and perinatal outcomes of women with COVID19 pneumonia: A preliminary analysis. AJRAm J Roentgenol. 2020;215(1):127-32. PMid:32186894

4. Chen H, Guo J, Wang C, Luo F, Yu X, Zhang W, et al. Clinical 
characteristics and intrauterine vertical transmission potential of COVID-19 infection in nine pregnant women: A retrospective review of medical records. Lancet. 2020;395(10226):809-15. https://doi.org/10.1016/s0140-6736(20)30360-3

PMid:32151335

5. Thomson G, Ebisch-Burton K, Flacking R. "Shame if you do-shame if you don't: Women's experiences of infant feeding. Matern Child Nutr. 2015;11(1):33-46. https://doi.org/10.1111/ mcn.12148

PMid:25138617

6. World Health Organization. Psychosocial Support for Pregnant Women and for Families with Microcephaly and other Neurological Complications in the Context of Zika Virus: Interim Guidance for Health-care Providers. Geneva: World Health Organization; 2016.

7. Alipour Z, Kheirabadi GR, Kazemi A, Fooladi M. "The most important risk factors affecting mental health during pregnancy: A systematic review. East Mediterr Health J. 2018;24(6):549-59. https://doi.org/10.26719/2018.24.6.549 PMid:30079950

8. Shapiro GD, Fraser WD, Frasch MG, Seguin JR. Psychosocial stress in pregnancy and preterm birth: Associations and mechanisms. J Perinat Med. 2013;41(6):631-45. https://doi. org/10.1515/jpm-2012-0295

PMid:24216160

9. Qiao Y, Wang J, Li J, Wang J. Effects of depressive and anxiety symptoms during pregnancy on pregnant, obstetric and neonatal outcomes: A follow-up study. J Obstet Gynecol. 2012;32(3):23740. https://doi.org/10.3109/01443615.2011.647736 PMid:22369395

10. Zeng Y, Cui Y, Li J. Prevalence and predictors of antenatal depressive symptoms among Chinese women in their third trimester: A cross-sectional survey. BMC Psychiatry. 2015;15:66. https://doi.org/10.1186/s12888-015-0452-7

PMid:25879965

11. Elovainio $M$, Hakulinen $C$, Pulkki-Råback $L$, Virtanen $M$, Josefsson $K$, Jokela $M$, et al. Contribution of risk factors to excess mortality in isolated and lonely individuals: An analysis of data from the UK Biobank cohort study. Lancet Public Health. 2017;2(6):e260-6. https://doi.org/10.1016/ s2468-2667(17)30075-0

PMid:28626828

12. Kalcev G, Preti A, Orrù G, Carta MG. Mental health and the COVID-19 pandemic: A call for action. Open Public Health J 2020;13:411-2. https://doi.org/10.2174/1874944502013010411

13. Lebel C, MacKinnon A, Bagshawe M, Tomfohr-Madsen L, Giesbrecht G. Elevated Depression and Anxiety among Pregnant Individuals during the COVID-19 Pandemic (PsyArXiv Preprints); 2020. https://doi.org/10.31234/osf.io/gdhkt

14. Duranku F, Aksu E. Effects of the COVID-19 pandemic on anxiety and depressive symptoms in pregnant women: $A$ preliminary study. J Matern Fetal Neonatal Med. 2020;1:1-7. PMid:32419558

15. Saccone G, Florio A, Aiello F, Venturella R, De Angelis MC, Locci M, et al. Psychological impact of coronavirus disease 2019 in pregnant women. Am J Obstet Gynecol. 2020;223(2):293-5. https://doi.org/10.1016/j.ajog.2020.05.003

PMid:32387321

16. Huntley BJ, Huntley ES, Di Mascio D, Chen T, Berghella V, Chauhan SP. Rates of maternal and perinatal mortality and vertical transmission in pregnancies complicated by severe acute respiratory syndrome coronavirus 2 (SARS-Co-V-2) infection: A systematic review. Obstet Gynecol. 2020;136:30312. https://doi.org/10.1097/aog.0000000000004010 PMid:32516273

17. Yang $Y$, Li W, Zhang Q, Zhang L, Cheung T, Xiang YT. Mental health services for older adults in China during the COVID19 outbreak. Lancet Psychiatry. 2020;7(4):e19. https://doi. org/10.1016/s2215-0366(20)30079-1

PMid:32085843

18. Brooks SK, Webster RK, Smith LE, Woodland L, Wessely S, Greenberg N, et al. The psychological impact of quarantine and how to reduce it: Rapid review of the evidence. Lancet. 2020;395(10227):912-20. https://doi.org/10.1016/ s0140-6736(20)30460-8

19. National Institute for Health and Care Excellence. Antenatal and Postnatal Mental Health. United Kingdom: National Institute for Health and Care Excellence; 2016. https://doi.org/10.1093/ ww/9780199540884.013.u13729 